\title{
Remote monitoring for heart failure using implantable devices: a systematic review, meta-analysis, and meta-regression of randomized controlled trials
}

\author{
Alexander G. Hajduczok ${ }^{1}$ (1) $\cdot$ Samer N. Muallem ${ }^{1} \cdot$ Matthew S. Nudy $^{2} \cdot$ Ami L. DeWaters $^{1} \cdot$ John P. Boehmer ${ }^{2}$
}

Accepted: 14 July 2021 / Published online: 24 September 2021

(c) The Author(s), under exclusive licence to Springer Science+Business Media, LLC, part of Springer Nature 2021

\begin{abstract}
In heart failure (HF) patients, remote monitoring using implantable devices may be used to predict and reduce HF exacerbations and mortality. Data from randomized controlled trials (RCTs) was assessed to determine the effectiveness of implantable remote monitoring on the improvement of outcomes in HF patients. A systematic review and meta-analysis of RCTs testing remote monitoring versus standard of care for management of HF patients was performed. Primary endpoints were all-cause mortality and a composite of cardiovascular (CV) and HF hospitalizations. Rate ratios (RRs) and 95\% confidence intervals (CI) were calculated. A secondary analysis tested for heterogeneity of treatment effect (HTE) comparing right ventricular/pulmonary pressure monitoring versus impedance-based monitoring on hospitalization. A regression analysis was performed using the mean follow-up time as the moderator on each primary endpoint. Eleven RCTs $(n=6196)$ were identified with a mean follow-up of 21.9 months. The mean age and reported ejection fraction were 64.1 years and $27.7 \%$, respectively. Remote monitoring did not reduce mortality (RR 0.89 [95\% CI 0.77, 1.03]) or the composite of CV and HF hospitalizations (RR 0.98 [0.81, 1.19]). Subgroup analysis found significant HTE for hospitalizations between those studies that used right ventricular/pulmonary pressure monitoring versus impedance-based monitoring $\left(I^{2}=87.1 \%, \mathrm{chi}^{2}=7.75\right.$, $p=0.005)$. Regression analysis found no relationship between the log rate ratio of remote monitoring's effect on mortality, $\mathrm{CV}$ hospitalization or HF hospitalization, and mean follow-up time. Compared to standard of care, remote monitoring using implantable devices did not reduce mortality, CV, or HF hospitalizations. However, right ventricular/pulmonary pressure monitoring may reduce HF hospitalizations, which will need to be explored in future studies.
\end{abstract}

Keywords Heart failure $\cdot$ Remote monitoring $\cdot$ Implantable devices $\cdot$ Systematic review $\cdot$ Meta-analysis $\cdot$ Randomized controlled trials

\section{Highlights}

- Standard of care in heart failure outpatient monitoring is centered on patient-reported symptoms.

- New methods of monitoring physiologic markers with implantable devices such as cardiac resynchronization therapy device and pulmonary artery pressure sensors have been developed.

- Several randomized controlled trials (RCTs) have been conducted examining whether use of remote monitoring has an effect on mortality and hospitalizations.

- This systematic review and meta-analysis examined 11 RCTs and showed no significant effect with implantable remote monitoring on mortality, heart failure (HF) hospitalizations, or cardiovascular hospitalizations when compared to standard of care.

- A decrease in HF hospitalizations was observed in RCTs using implantable continuous cardiac/pulmonary artery pressure monitoring compared to thoracic impedance-based monitoring strategies.

Extended author information available on the last page of the article

\author{
Abbreviations \\ $\mathrm{HF} \quad$ Heart failure \\ CV Cardiovascular \\ RCT Randomized controlled trial \\ CRT-D Cardiac resynchronization therapy defibrillator \\ ICD Implantable cardioverter-defibrillator
}

\section{Introduction}

Heart failure (HF) is a complex clinical syndrome that affects over 26 million people worldwide [1,2]. The disease poses a tremendous strain on the current medical system due to frequent rehospitalizations, accounting for over 1 million annual hospital admissions [3]. In patients with HF, the standard of care for surveillance of chronic disease has been to monitor symptoms and maintain frequent outpatient 
follow-up with patient-initiated phone calls if symptoms worsen. Outpatient diuretic regimens are adjusted in real time to help reduce volume overload and improve heart failure symptoms. Early follow-up for HF hospitalizations, within 7 days of discharge, has been associated with a lower 30-day readmission rate, suggesting a benefit for closer monitoring of HF patients [4]. Continuous remote monitoring of specific metrics in HF patients may lead to earlier interventions and therefore improved outcomes $[5,6]$.

Randomized controlled trials (RCTs) have tried to replicate this close follow-up and monitoring technique using telemonitoring that transmits metrics such as blood pressure, weights, and symptoms $[5,7,8]$. Individual studies have not shown a large benefit in reducing HF hospitalizations using telemonitoring, but meta-analysis of these RCTs suggests that there may be a role for telemonitoring in reducing mortality and HF hospitalizations [8-10].

In addition to telemonitoring, a new method of surveillance for disease severity has emerged in the form of remote monitoring of implantable devices [7, 11]. Such devices, which include implanted cardiac defibrillators, dual chamber pacemakers, cardiac resynchronization therapy devices, and implantable hemodynamic pressure sensors, can measure physiologic parameters such as intrathoracic impedance, tachyarrhythmias, and intracardiac or pulmonary artery pressure, thereby providing actionable data to guide therapy [1, $5,11]$.

The physiologic measures these devices are able to monitor theoretically correlate with heart failure exacerbation states [11], but their utilization for heart failure management with mortality benefit remains to be seen [12]. Ongoing research is being conducted to determine whether monitoring of this physiologic data can be utilized to make medication adjustments in the outpatient setting and control heart failure symptoms to prevent hospitalization [13]. This was based on previous observational studies that utilized hemodynamic-based heart failure management strategies, which improved New York Heart Association (NYHA) class and decreased HF hospitalizations [14, 15].

Due to the inconclusive RCT and meta-analysis data about the effectiveness of telemonitoring, additional research has been performed to assess objective measures that correlate with worsening HF, specifically via implantable remote monitoring devices [12, 13, 16-28]. Whereas the current standard of care relies on patient-reported symptoms for intervention, utilization of remote monitoring would give more objective clinical data to help drive management, and may improve outcomes $[11,12,17]$. Given the burden of heart failure hospitalizations on the individual patient as well as the larger healthcare system, prevention of HF exacerbation is a critical goal [1-3].

We aimed to review and analyze the current literature on invasive remote monitoring in HF patients to assess whether remote monitoring of physiologic markers of disease severity leads to a reduction in mortality, cardiovascular-related hospitalization, or heart failure hospitalization rates when compared to standard of care (routine outpatient follow-up).

\section{Methods}

\section{Literature search strategy, selection criteria, and outcomes of interest}

The Preferred Reporting Items for Systematic Reviews and Meta-Analysis (PRISMA) document was used as a guide and followed [29, 30]. Medline/PubMed, Embase, and Cochrane databases were searched for English language studies published between January 1, 1990 and August 9, 2019. Studies of interest included prospective randomized controlled trials (RCTs) testing remote monitoring versus control (standard of care) in adult ( $>18$ years) patients with HF and analyzed various outcomes using implantable remote monitoring modalities that were able to directly or indirectly report hemodynamic information. "Standard of care" was defined as usual or routine follow-up for heart failure, based on clinician discretion, including outpatient visits and bloodwork, but not using remote monitoring or electronic transmission of data to guide management. Outcomes of interest for this analysis included all-cause mortality and heart failure hospitalization. If an RCT did not report an outcome of interest, it was not included in this analysis. Remainder of inclusion criteria included subjects with New York Heart Failure (NYHA) HF classes I-IV and use of an implantable remote monitoring device that had remote monitoring capabilities (intracardiac pressures, pulmonary artery pressures, thoracic impedance, continuous arrhythmia monitoring, or a combination of these parameters). Exclusion criteria included studies that only utilized remote telemonitoring that did not transmit information from an implantable device or studies that only transmitted arrhythmia data from ICD or CRT-D devices. Initial keywords that were used included "Heart Failure, remote monitoring, wearable technology, heart sensor, implantable hemodynamic monitoring, randomized controlled trial, mortality, and hospital stay."

\section{Data extraction and quality assessment}

Data extraction was done in two phases: a practical review and a methodological review. In the practical review, the title and abstract of each of the 1604 articles retrieved in the search were reviewed independently for inclusion by a team of two reviewers (AH and SM), after removing duplicates. Gray literature was also searched, with revealed no additional articles. 
Disagreements were resolved by consensus and resulted in 105 articles selected for full review. In the full-text review, inclusion/exclusion criteria were applied again, yielding 25 articles eligible for methodological review. These 25 studies were then further narrowed to 11 randomized controlled trials (RCTs) after eliminating duplicate datasets and studies that did not have the primary outcomes of mortality and cardiovascular (CV) hospitalizations or HF hospitalizations, or included the proper forms of remote monitoring (Fig. 1).

Data were independently recorded in a standardized manner for each RCT. Supplemental appendices were also searched if data were incomplete. Any inconsistencies were reassessed by all parties until the data were determined to be accurate.

All included studies were graded for bias using the Cochrane Handbook for Systematic Review of Interventions by two authors (AH and SM). Bias was assessed on predetermined criteria including random sequence generation, allocation concealment, blinding of participants and personnel, blinding of outcome assessment, incomplete outcome data, selective reporting, and other (i.e., predetermined outcome of trial, financial consideration) $[31,32]$.

\section{Statistical analysis}

The primary analysis was performed using the Mantel-Haenszel method, and summary rate ratios (RRs) and $95 \%$ confidence interval (CI) were calculated using a random effect model for each endpoint. Total patient-years were calculated using trial duration and number of patients in each arm of the included studies. Examination of heterogeneity across the RCTs was assessed using $Q$ statistics and $I^{2}$ [33]. The 95\% CIs were estimated using a binominal distribution. A sensitivity analysis was performed which excluded the 3 studies that only reported all-cause hospitalizations [23, 24, 26] and included those that reported HF hospitalization specifically [17, 19-22, 25, 27, 34]. A random effect model was utilized given the inherent variability in patient population, device types, remote monitoring protocols, variation in control arm oversight, and follow-up times of the included studies. Publication bias was visually assessed using funnel plots.

An exploratory subgroup analysis was performed comparing implanted right ventricular/pulmonary pressure monitoring versus impedance-based monitoring on each hospitalization outcome. This was also performed for clinician-based versus patient-based alerts and reported for both hospitalization outcomes. In both
Fig. 1 Preferred Reporting Items for Systematic Reviews and Meta-Analysis (PRISMA) flow diagram of systematic review, which represents the number of studies screened, assessed, and included in the meta-analysis. One thousand six hundred four references were reviewed, yielding a total of 11 randomized controlled trials for final analysis
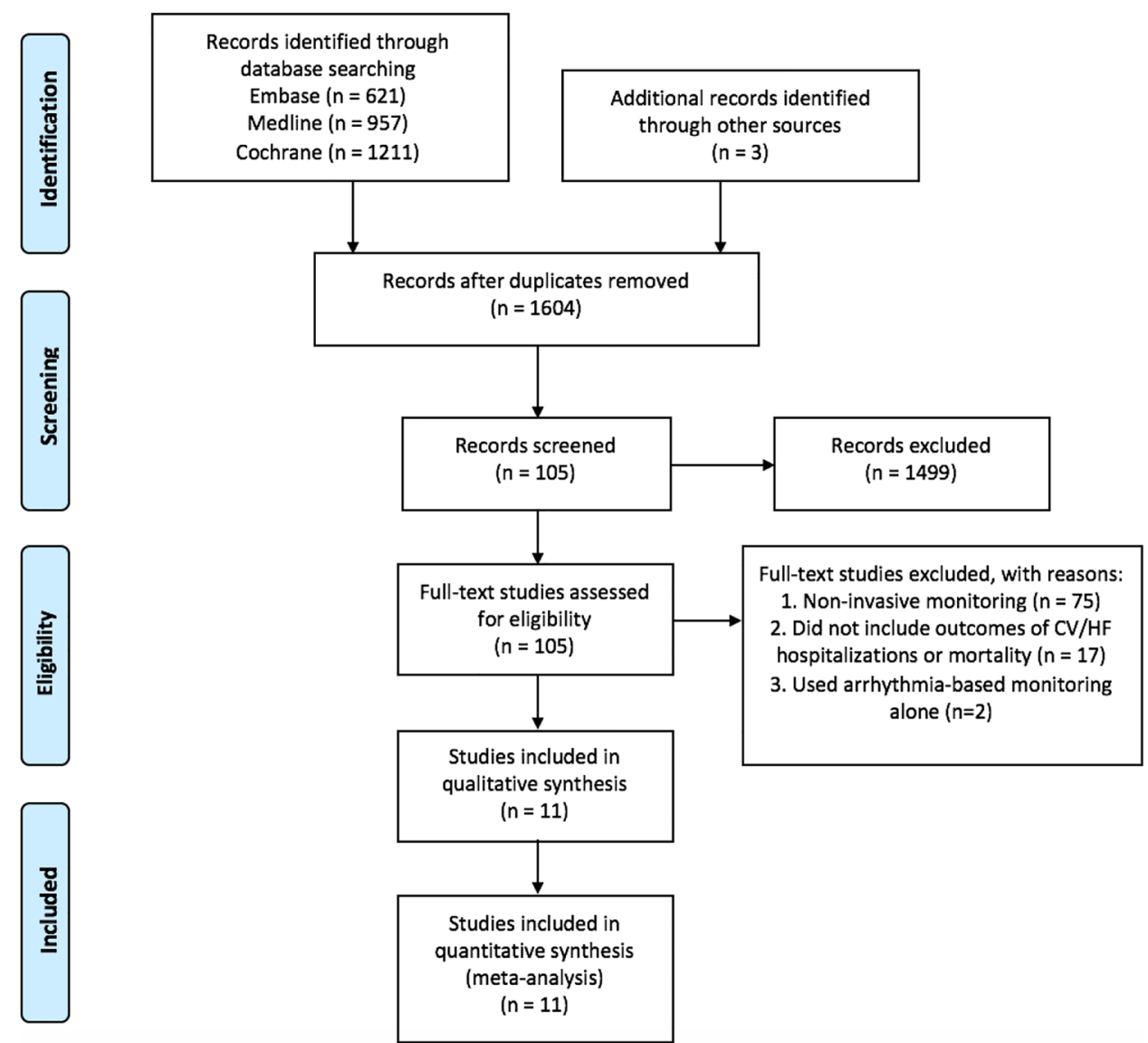
cases, summary RRs with 95\% CI were calculated. Each subgroup was assessed for heterogeneity, and the test for subgroup differences was performed using $\mathrm{chi}^{2}$ and $I^{2}$ tests to assess for heterogeneity of treatment effect (HTE) [35].

A random effect meta-regression was performed using the mean follow-up time in months of each RCT as the moderator to determine if this continuous variable contributed to the heterogeneity in the mortality, HF or CV hospitalization, and HF hospitalization outcome. Meta-regression linear graphs were created by plotting the moderator variable (mean follow-up time) on the $x$-axis and the treatment effect of remote monitoring on the $y$-axis (the log of the rate ratio of remote monitoring's treatment effect of mortality, $\mathrm{HF}$ or $\mathrm{CV}$ hospitalization, and HF hospitalization for each RCT). When interpreting meta-regression, the log of the rate ratio used is on the $y$-axis. A log value of zero corresponds to a rate ratio of one; a negative log value corresponds to a rate ratio less than one, and a positive log value corresponds to a rate ratio greater than one. Each circle in the regression represents an included RCT, and the size of the circle is proportional to the weight of each RCT in the regression. The darker line in the center is the regression line, and the outer lighter colored lines represent the $95 \%$ CI. The following statistical tests were used in the regression: $\mathrm{Tau}^{2}$ which estimates the true variance among trials, $I^{2}$ which represents the ratio of heterogeneity to total observed variation in the RCTs, and $R^{2}$ index which is the proportion of between study variance explained by the moderator (in this analysis mean follow-up time). Regression coefficients and 95\% CIs were calculated and describe how remote monitoring's treatment effect will change with a unit change in the moderator variable.

Statistical analyses were conducted, and forest plots were created with Review Manager (RevMan [Computer program]. Version 5.3. Copenhagen: The Nordic Cochrane Centre, The Cochrane Collaboration, 2014). The meta-regression was performed using Comprehensive Meta-Analysis Version 3, Biostat, Englewood, NJ, 2013.

\section{Results}

Eleven RCTs were identified comparing remote monitoring of implantable devices with hemodynamic monitoring capabilities to standard of care for heart failure management. These RCTs had a total of 6196 participants with weighted mean follow-up time of 21.9 months $(10,667$ patient-years of follow-up). The mean age and reported ejection fraction were 64.1 years and $27.7 \%$, respectively (Fig. 1 and Table 1). There was some variation in primary endpoints between the studies, with 8 measuring HF hospitalizations and the remaining 3 measuring the broader measure of $\mathrm{CV}$ hospitalizations, which were defined as any hospitalizations with a cardiovascular diagnosis as the reason for admission (Table 2). All 11 RCTs had mortality data included as either primary or secondary endpoints (Tables 1 and 2 and Appendix 1). When comparing remote monitoring to standard of care, there was no significant reduction in mortality ( $R R$ 0.89 [95\% CI 0.77-1.03]) or the composite of CV or HF hospitalizations (RR 0.98 [95\% CI 0.81-1.19]) (Figs. 2 and 3). Statistically significant heterogeneity existed among the RCTs analyzing CV or HF hospitalization $\left(I^{2}=90 \%\right.$, $\left.\mathrm{chi}^{2}=101.02, p<0.0001\right)$. Minimal, although statistically insignificant, heterogeneity existed amongst the RCTs when analyzing all-cause mortality $\left(I^{2}=7 \%, \mathrm{chi}^{2}=10.7, p=0.38\right)$. On visual evaluation of the funnel plot, there was no evidence of publication bias for both of the measured primary outcomes (Supplementary Figs. 3, 4).

Sensitivity analysis examining exclusively HF hospitalizations with data from 8 of the 11 RCTs included revealed no significant reduction in HF hospitalizations in the remote monitoring group compared to control (RR 0.97 [95\% CI $0.74-1.24])$. Statistically significant heterogeneity was observed among these 8 RCTs $\left(\mathrm{chi}^{2}=64.9, p<0.0001\right.$, $I^{2}=89 \%$ ) (Fig. 4).

Given the significant heterogeneity observed when analyzing the hospitalization outcome, exploratory subgroup analyses were performed based on type of remote monitoring and alert type (clinician versus patient). Subgroup analysis showed that there was a statistically significant difference in the composite of CV or HF hospitalizations and HF hospitalizations alone when comparing studies using implanted right ventricular/pulmonary pressure monitoring versus impedance-based monitoring, favoring the intracardiac pressure monitoring (RR 0.75 [95\% CI 0.59-0.95] vs. RR 1.10 [95\% CI 0.96-1.26]) and test for subgroup differences: $I^{2}=87.1 \%$, chi ${ }^{2}=7.75, p=0.005$ (Fig. 5, Supplemental Fig. 5). When RCTs were stratified by alert type (clinician versus patient alerts), there was an increase in both $\mathrm{CV}$ or HF hospitalizations and HF hospitalizations alone when studies used devices with patient alerts (RR 1.55 [95\% CI 1.28-1.89]) compared to clinician alerts (RR 0.91 [95\% CI $0.75-1.09])$ and test for subgroup differences: $I^{2}=93.7 \%$, chi $^{2}=15.87, p<0.0001$ (Fig. 6, Supplemental Fig. 6).

The overall risk of bias in the included RCTs was judged to be low, as 11/88 (12.5\%) of the domains were graded as moderate or high (Supplementary Table 1).

The meta-regression analysis found no statistically significant relationship between the log rate ratio of remote monitoring's effect on mortality and the mean follow-up time $\left[\mathrm{Tau}^{2}=0.01, I^{2}=17.1 \%\right.$ and $R^{2}=0.00$, regression coefficient $=0.004$ (95\% CI $-0.02-0.03)$ ] (Fig. 7). In addition, there was no statistically significant linear relationship between the log rate ratio of remote monitoring's effect on CV or HF hospitalization and mean follow-up time $\left[\mathrm{Tau}^{2}=0.07, I^{2}=78 \%\right.$ and $R^{2}=0.00$, regression 


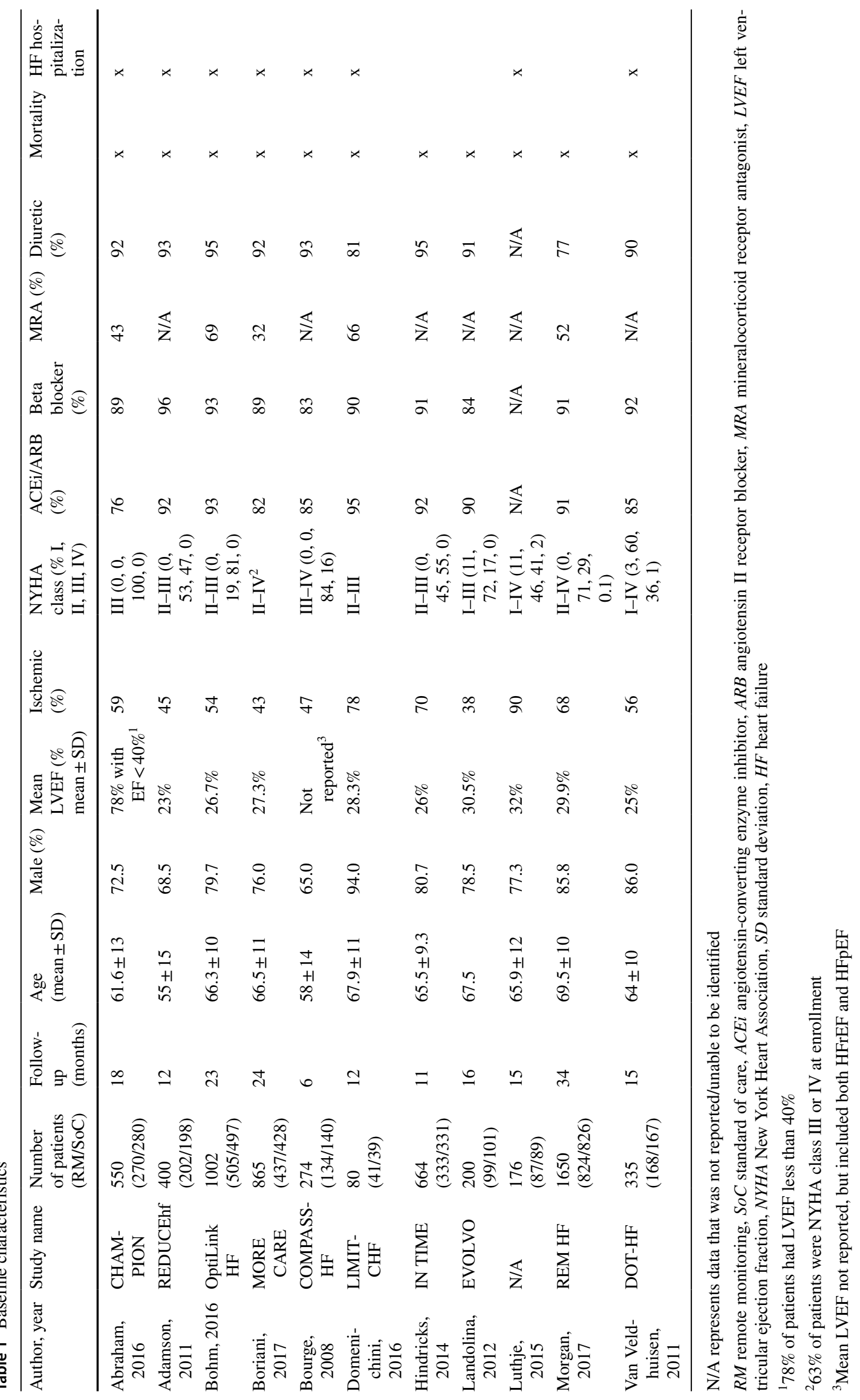




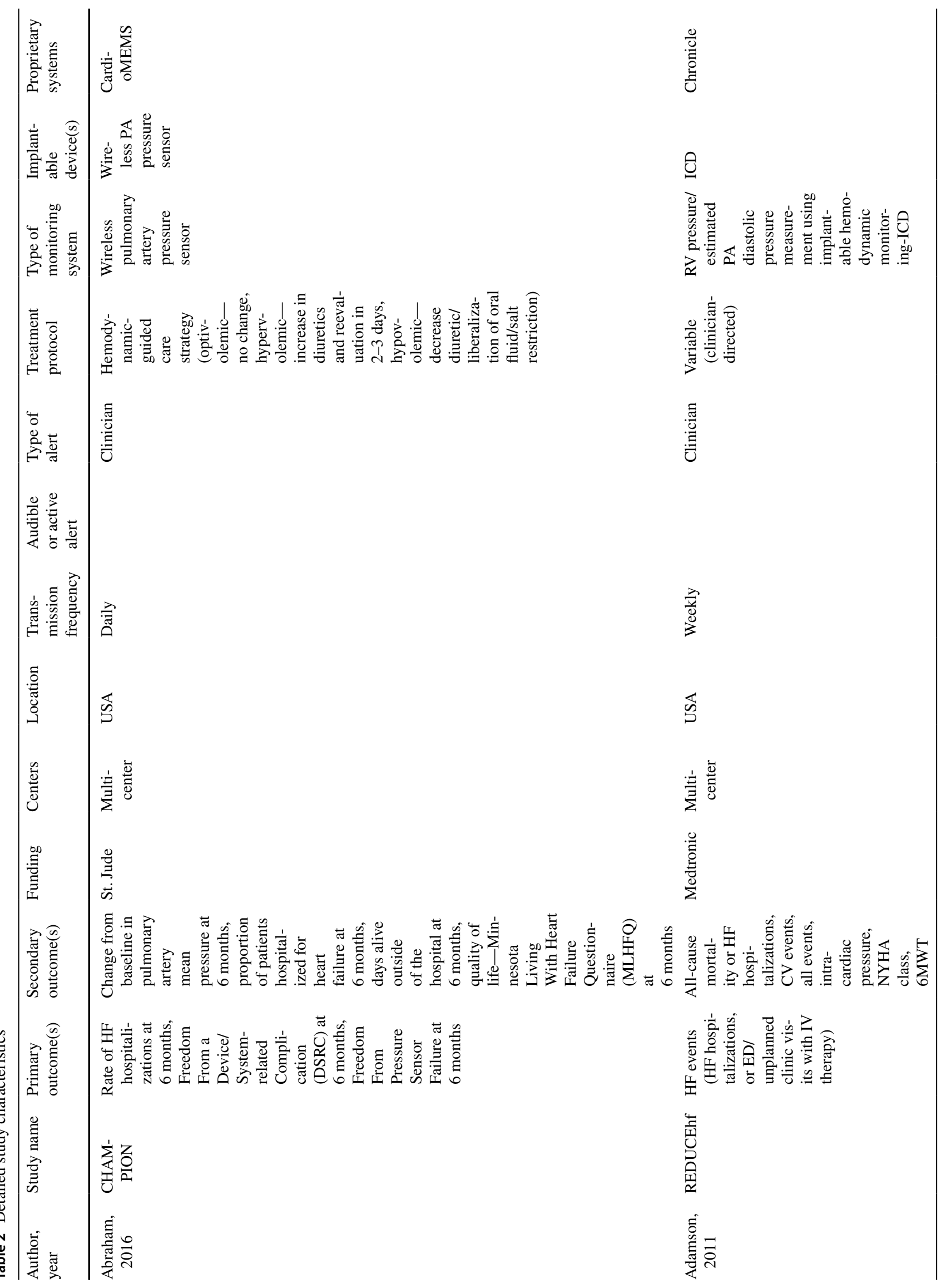




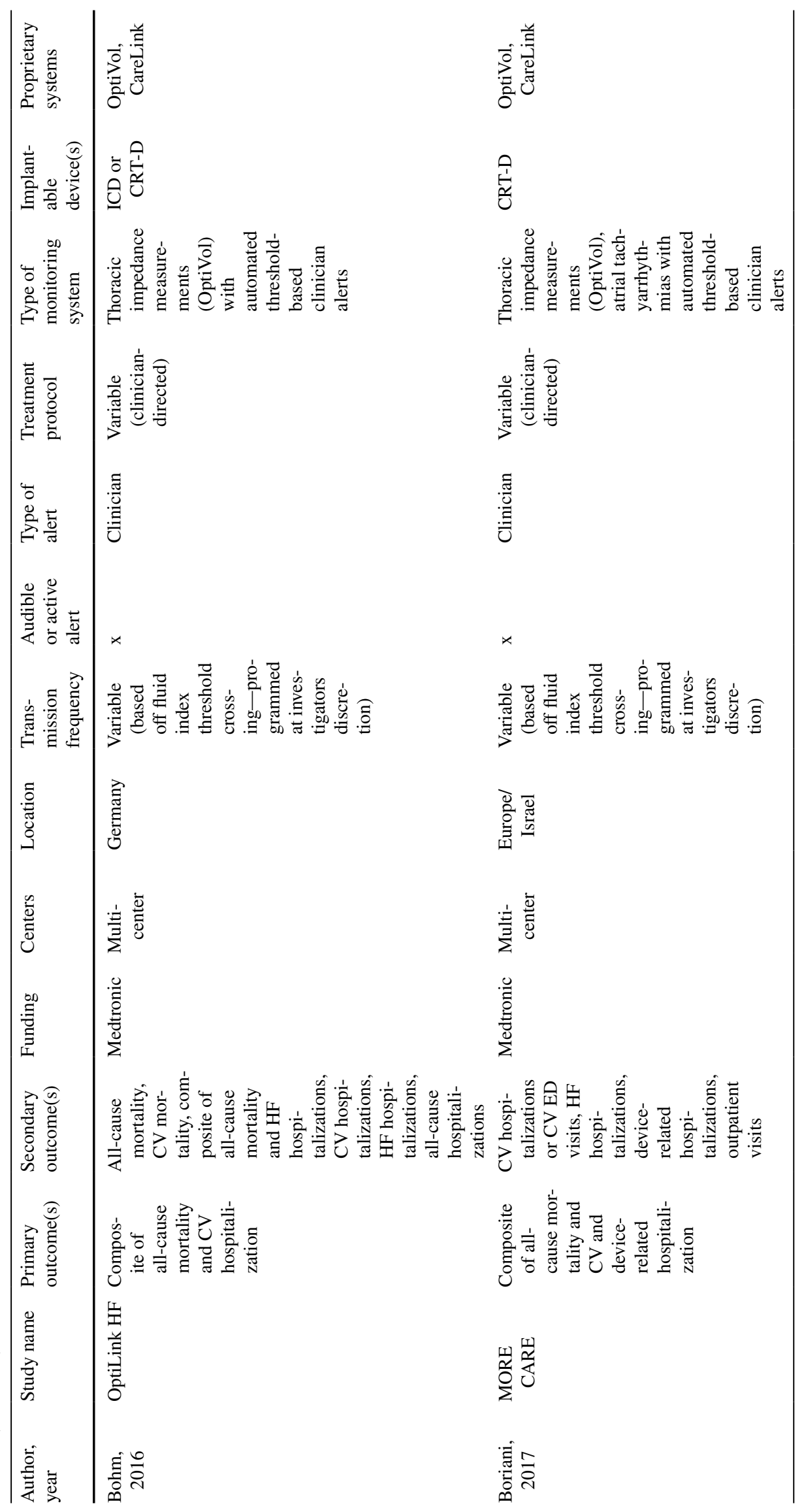




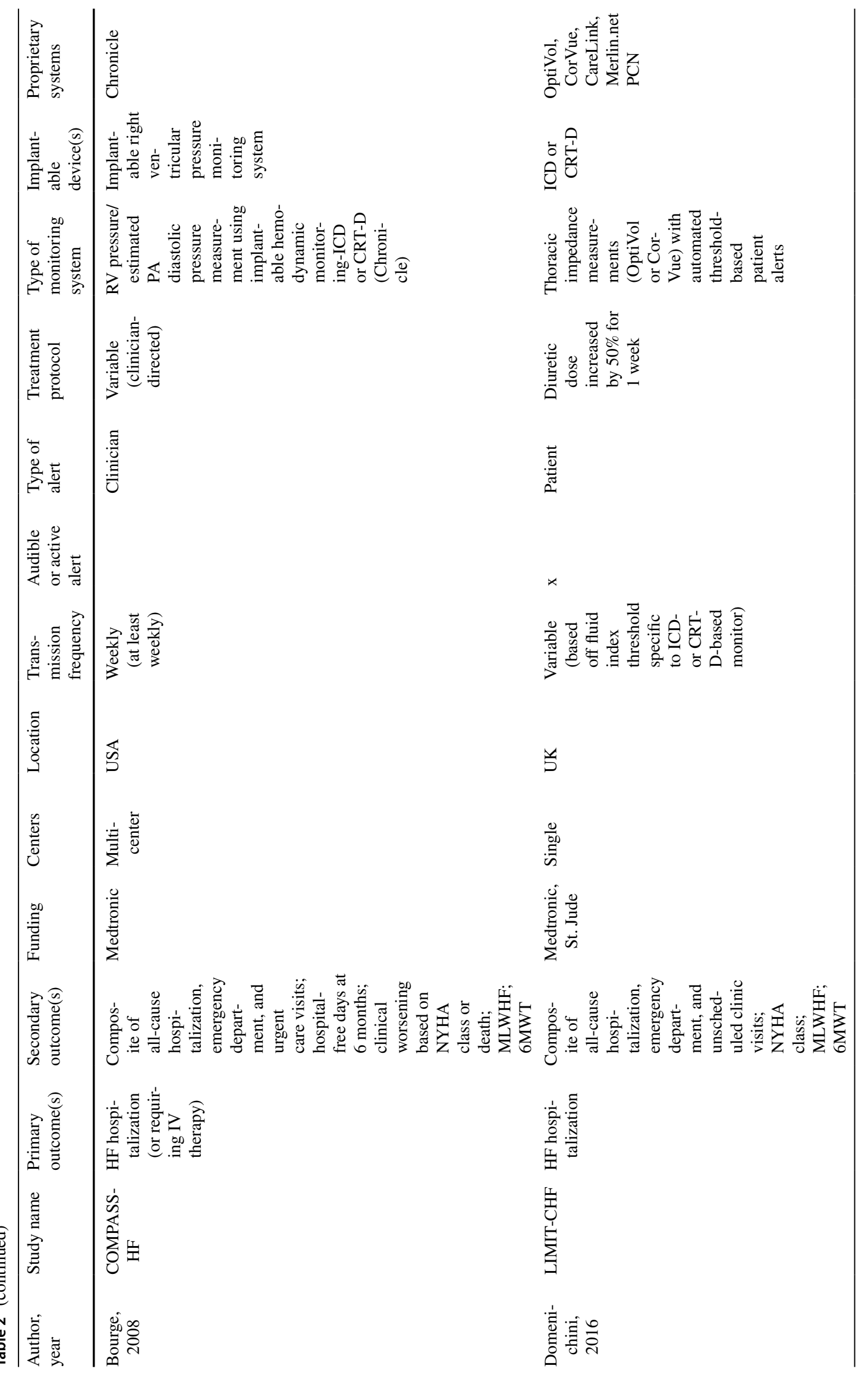




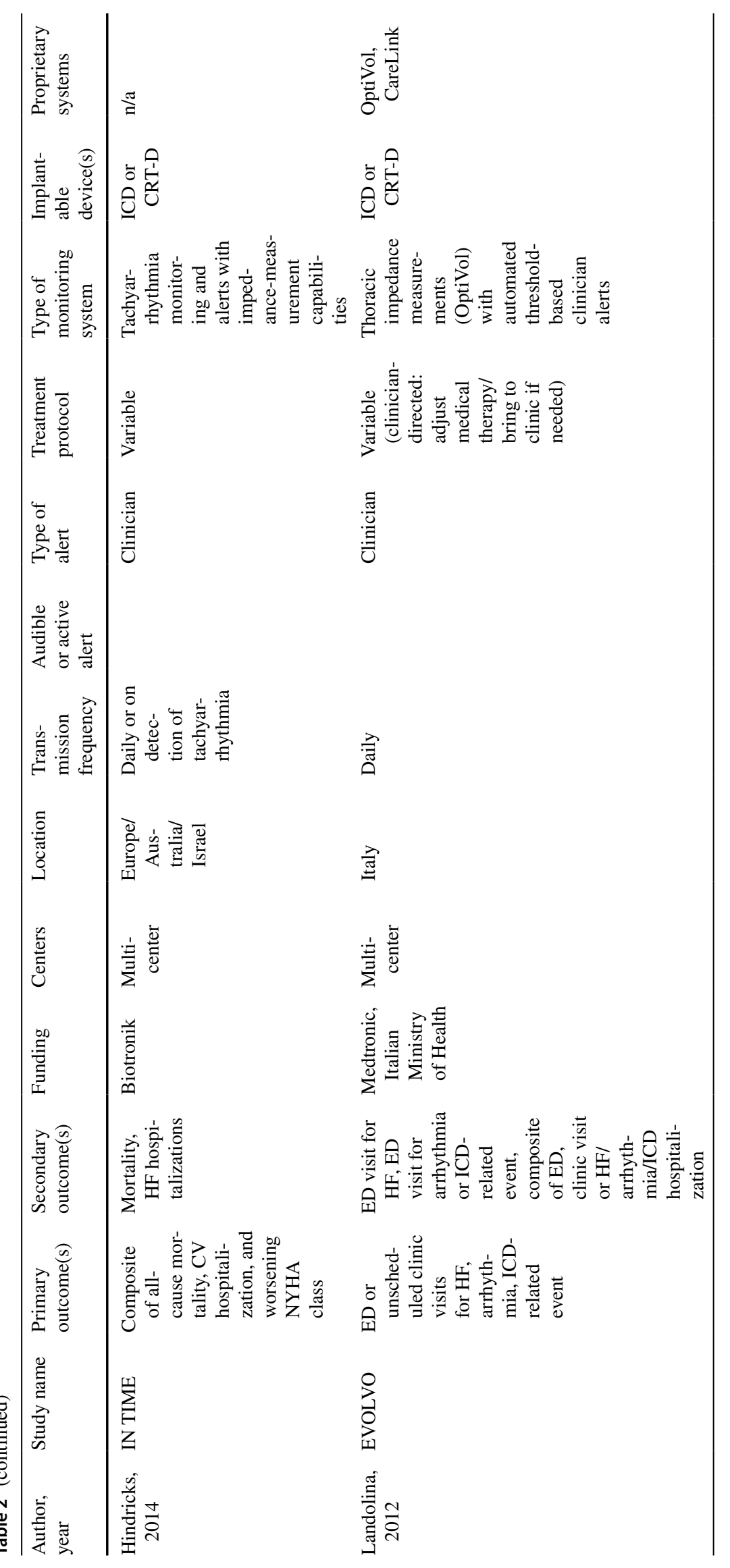




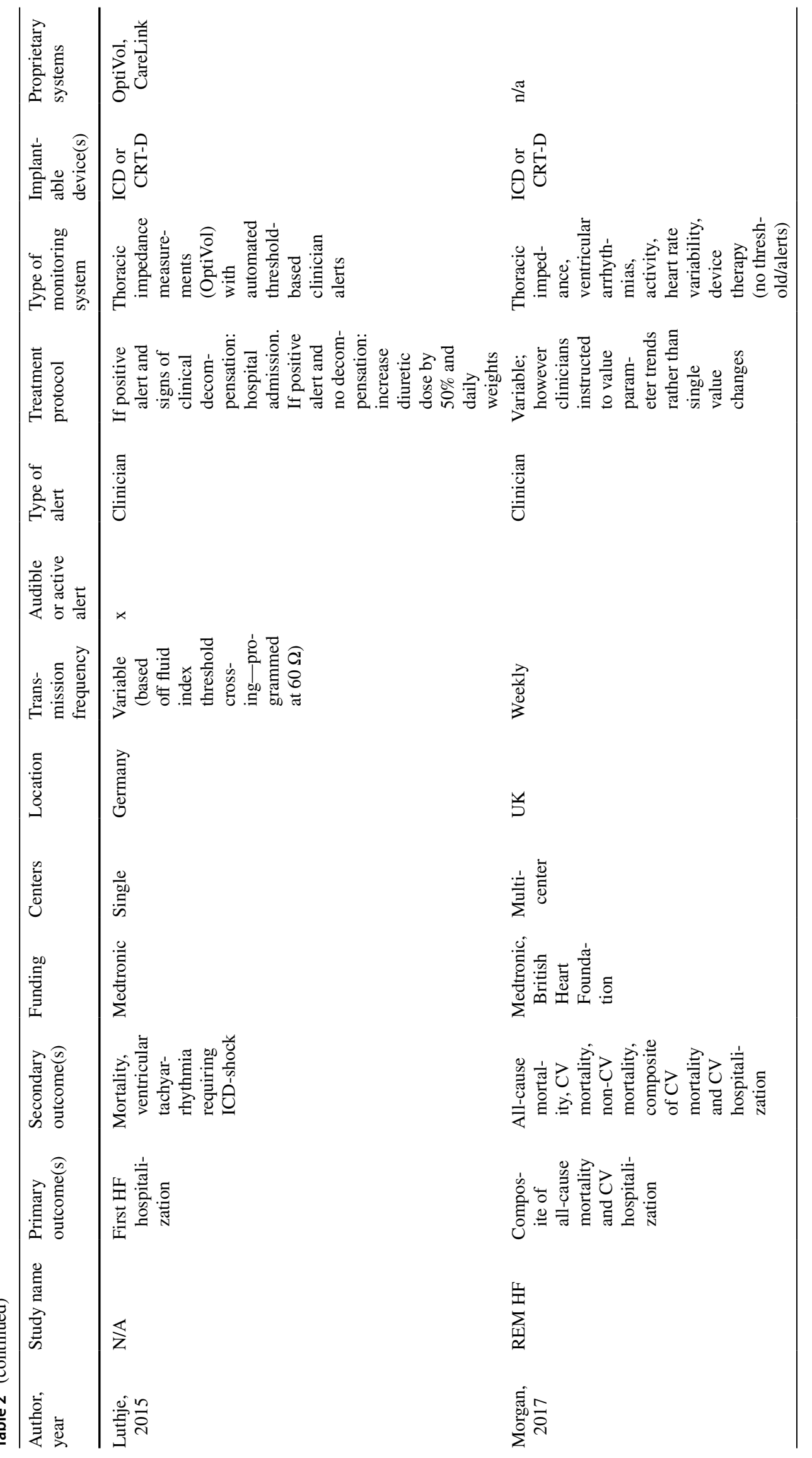




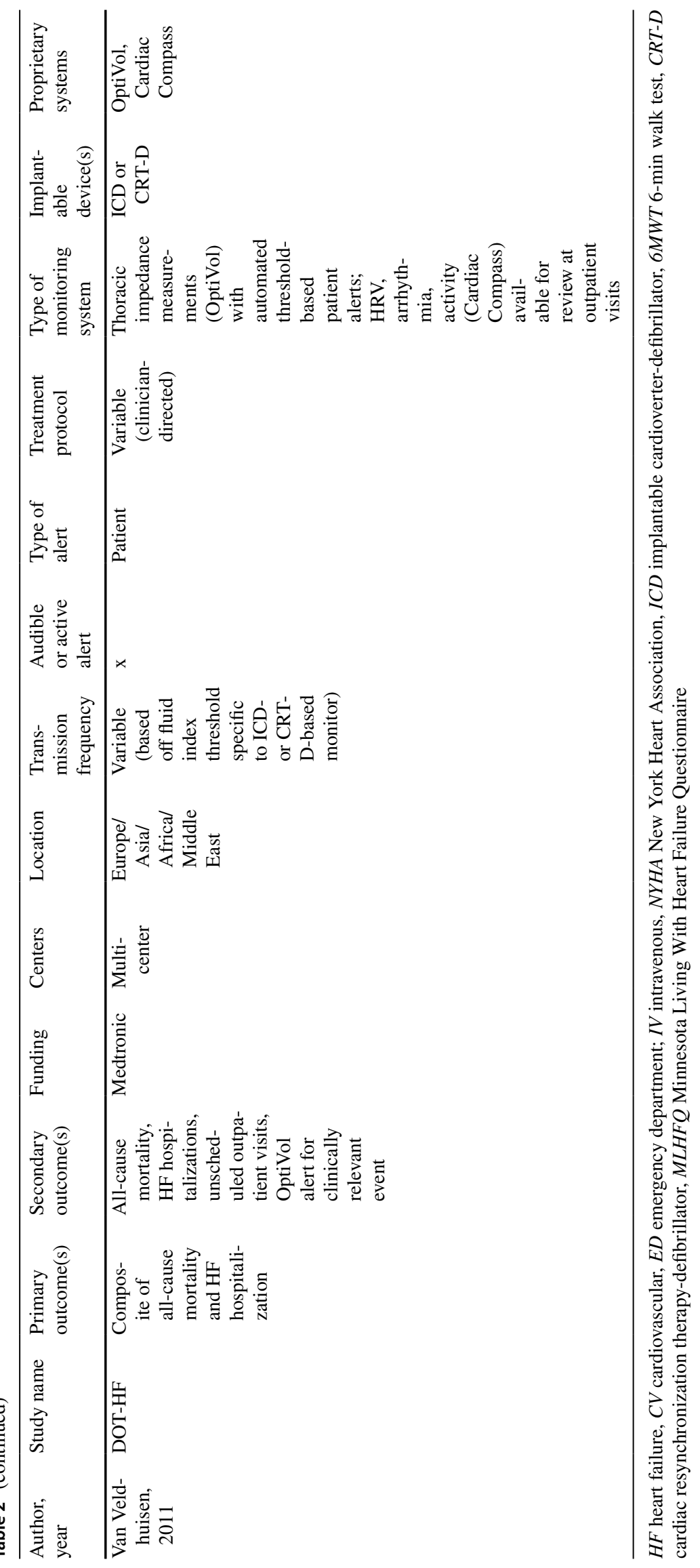




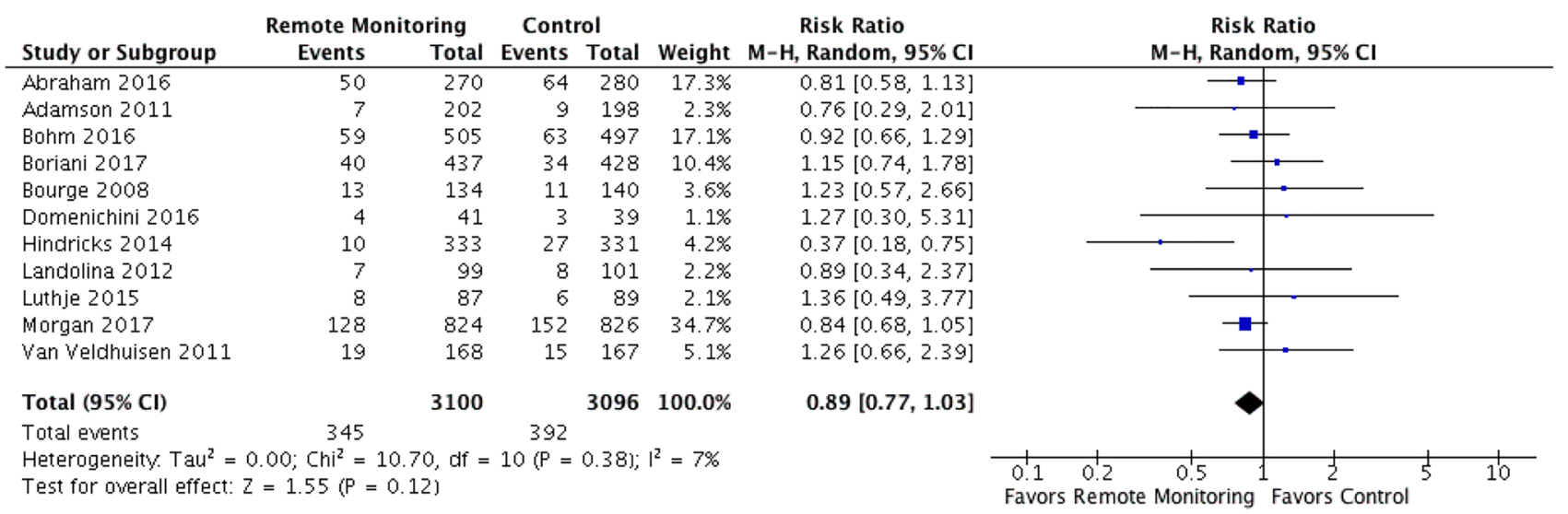

Fig. 2 All-cause mortality. Forest plot showing rate ratio and 95\% confidence for each endpoint among HF patients randomized to remote monitoring versus standard of care. When comparing remote monitoring to standard of care, there was no significant reduction in

coefficient $=0.01(95 \%$ CI $-0.01-0.04)]$ (Supplementary Fig. 1). Also, the meta-regression analysis found no significant relationship between the log rate ratio of remote monitoring's effect on HF hospitalization and the mean follow-up time $\left[\mathrm{Tau}^{2}=0.09, I^{2}=79.9 \%\right.$ and $R^{2}=0.1$, regression coefficient $=0.02(95 \% \mathrm{CI}-0.022-0.063)]$ (Fig. 5).

\section{Discussion}

Compared to standard of care, remote monitoring of physiologic parameters using implantable devices did not have a significant reduction in mortality or in the composite of $\mathrm{CV}$ or HF hospitalizations in patients with $\mathrm{HF}$ in the 11 RCTs included in this systematic review and meta-analysis. mortality (RR 0.89 [95\% CI 0.77-1.03]). Minimal, although statistically insignificant, heterogeneity existed among when analyzing allcause mortality $\left(I^{2}=7 \%\right.$, chi $\left.^{2}=10.7, p=0.38\right)$

Sensitivity analysis of HF hospitalizations alone, which was measured in 8 of the 11 RCTs, showed that remote monitoring had no significant reduction in HF hospitalizations. However, statistically significant heterogeneity was found in the studies measuring CV or HF hospitalizations leading to a subgroup analysis that revealed a statistically significant advantage in the composite of $\mathrm{CV}$ or HF hospitalizations and HF hospitalizations in studies using implanted right ventricular/pulmonary pressure monitoring when compared to studies using impedance-based monitoring.

The high heterogeneity among the hospitalization outcome can be due to multiple reasons: (1) only 11 studies met inclusion and exclusion criteria, with a relatively short mean total follow-up time (21.9 months) including only 6196 patients $(10,667$ patient-years); (2) variability

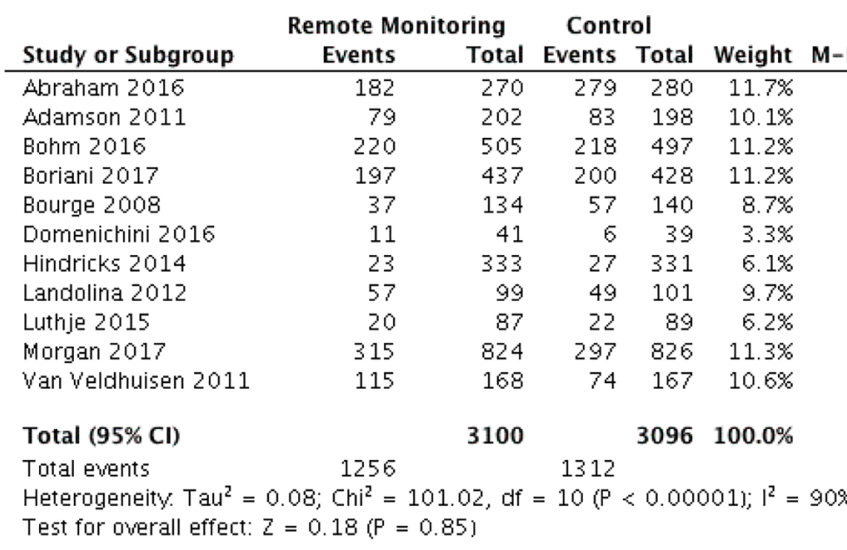

Fig. $3 \mathrm{CV}$ or HF hospitalizations. Forest plot showing rate ratio and 95\% confidence for each endpoint among HF patients randomized to remote monitoring versus standard of care. When comparing remote monitoring to standard of care, there was no significant reduction

\section{Risk Ratio Risk Ratio}

, Random, $95 \% \mathrm{Cl}$

$0.93[0.74,1.18]$

$0.99[0.86,1.14]$

$0.96[0.83,1.11]$

$0.68[0.48,0.95]$

$1.74[0.71,4.26]$

$0.85[0.50,1.45]$

$1.19[0.91,1.54]$

$0.93[0.55,1.58]$

$1.06[0.94,1.21]$

$1.54[1.27,1.88]$

$0.98[0.81,1.19]$

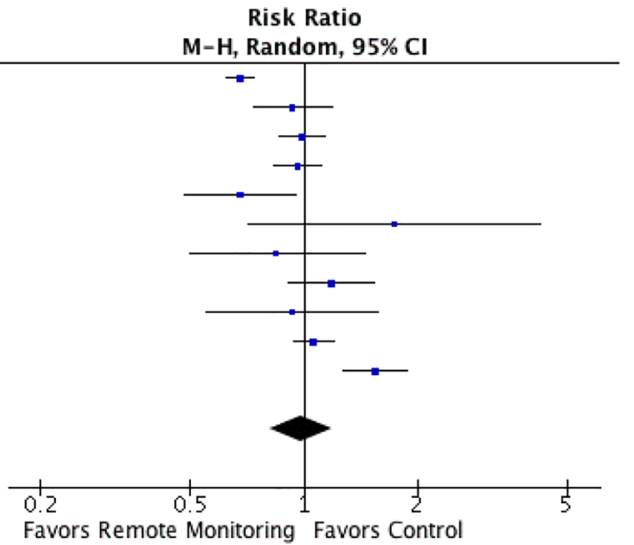

in the composite of CV or HF hospitalizations (RR 0.98 [95\% CI 0.81-1.19]). Statistically significant heterogeneity existed among the RCTs analyzing CV or HF hospitalization $\left(I^{2}=90 \%\right.$, chi $^{2}=101.02$, $p<0.0001)$ 


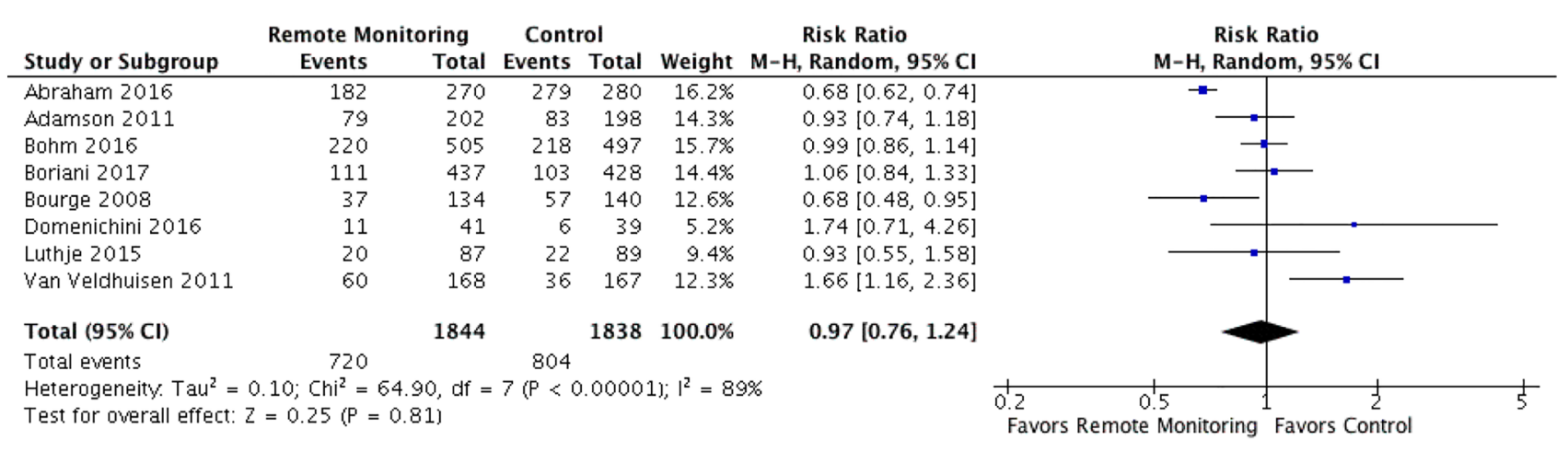

Fig. $4 \mathrm{HF}$ hospitalizations. Forest plot showing rate ratio and 95\% confidence for each endpoint among $\mathrm{HF}$ patients randomized to remote monitoring versus standard of care. Sensitivity analysis examining exclusively HF hospitalizations with data from 8 of the

in devices/measured parameters; and (3) variability in study protocols, including lack of a standardized treatment protocol. Multiple different devices, with proprietary data collection and transfer methods, were utilized among the RCTs, including CRT-D vs. ICD vs. pulmonary artery pressure sensor-based devices.

Any form of monitoring, but especially invasive methods as mentioned above and examined in this study, are not inexpensive; thus, it is critical to define whether these technologies are actually superior to the standard of care
11 RCTs included revealed no significant reduction in HF hospitalizations in the remote monitoring group compared to control (RR 0.97 [95\% CI 0.74-1.24]). Statistically significant heterogeneity was observed among these 8 RCTs $\left(\mathrm{chi}^{2}=64.9, p<0.0001, I^{2}=89 \%\right)$

for outpatient follow-up [11, 12]. Value-based care is becoming increasingly prevalent in outpatient cardiology, as it has become a goal to prevent rehospitalizations for HF [3]. This meta-analysis does not support invasive methods as a more "high value" option for patients with HF. We argue that the reason why this was not captured in the data from the 11 RCTs presented is because the metrics used may lack adequate sensitivity or specificity for the true pathophysiology of the heart failure disease state. In addition, patient-based alerts were associated

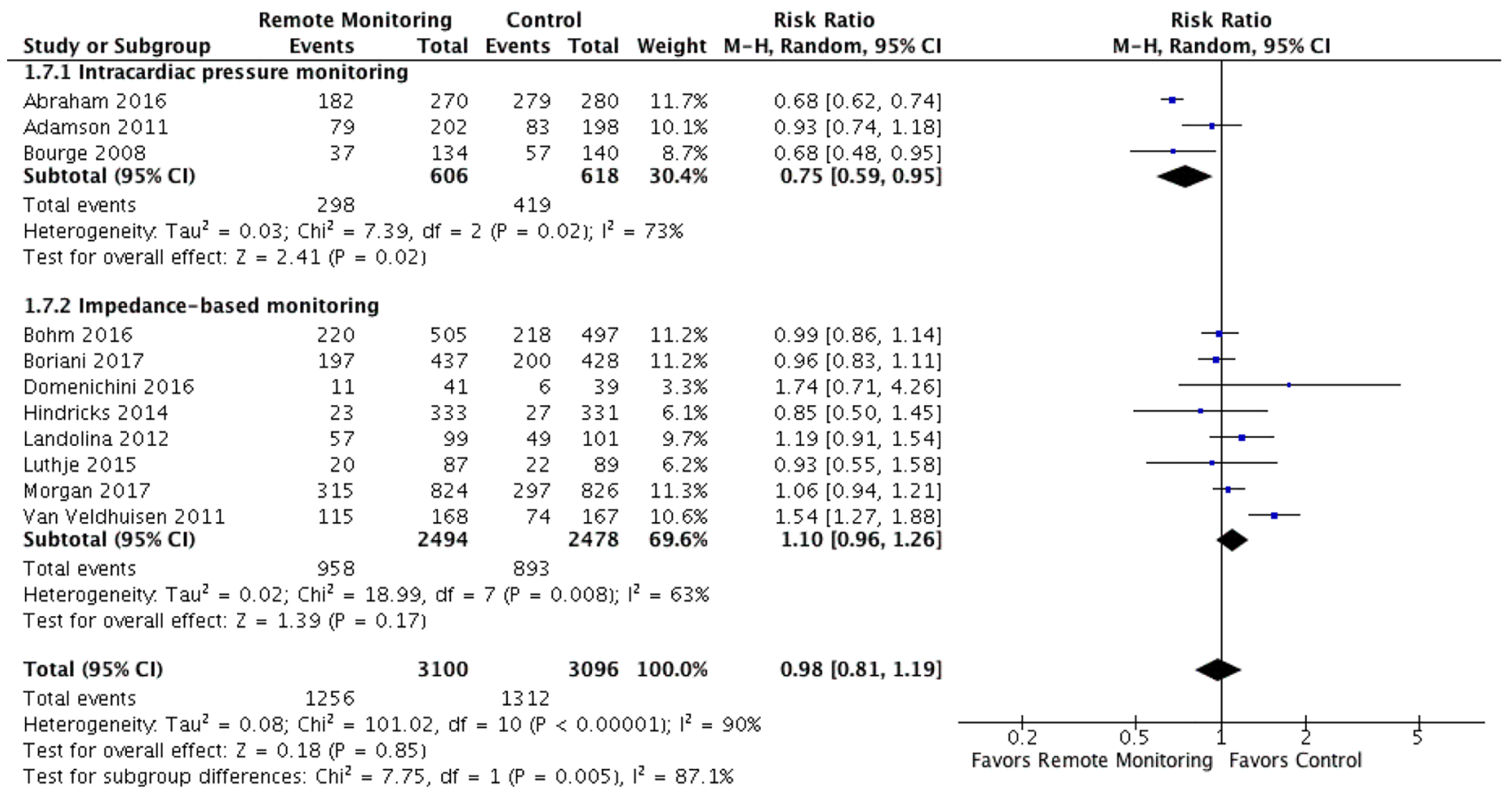

Fig. 5 Intracardiac pressure monitoring vs. thoracic impedance-based monitoring (subgroup analysis). Outcomes shown are the composite of HF or CV hospitalizations. Subgroup analysis showed that there was a statistically significant difference in the composite of $\mathrm{CV}$ or HF hospitalizations when comparing studies using implanted right ventricular/pulmonary pressure monitoring versus impedance-based monitoring, favoring the intracardiac pressure monitoring (RR 0.75 [95\% CI 0.59-0.95] vs. RR 1.10 [95\% CI 0.96-1.26]) and test for subgroup differences: $I^{2}=87.1 \%$, $\mathrm{chi}^{2}=7.75, p=0.005$ 


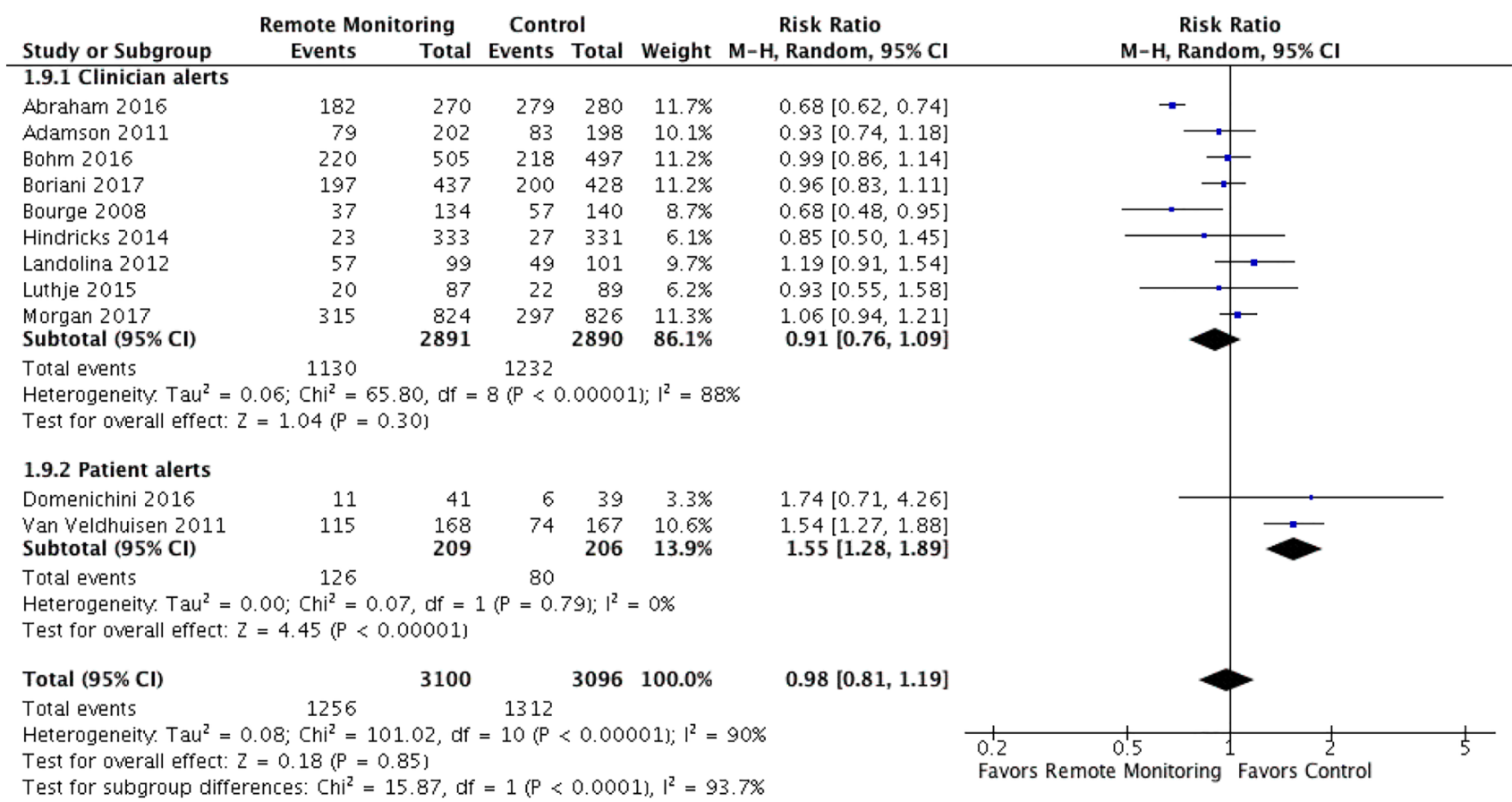

Fig. 6 Clinician vs. patient-based alerts (subgroup analysis). Outcomes shown are HF or CV hospitalizations. There was an increase in both $\mathrm{CV}$ or HF hospitalizations and HF hospitalizations alone when

with an increase in hospitalizations $[22,27]$. This is presumably due to biasing of symptomatology, leading to a lower threshold for HF admission if a remote monitoring device designed to detect HF exacerbations is in "alert." studies used devices with patient alerts (RR 1.55 [95\% CI 1.28-1.89]) compared to clinician alerts (RR 0.91 [95\% CI 0.76-1.09]) and test for subgroup differences: $I^{2}=93.7 \%, \mathrm{chi}^{2}=15.87, p<0.0001$

Clinician-based alerts did not individually increase hospitalizations. Therefore, this raises concern that these alerts could lead to an unnecessary increase in hospitalization (Table 2).

\section{Regression of Log Rate Ratio of Remote Monitoring's Treatment Effect on Mortality}

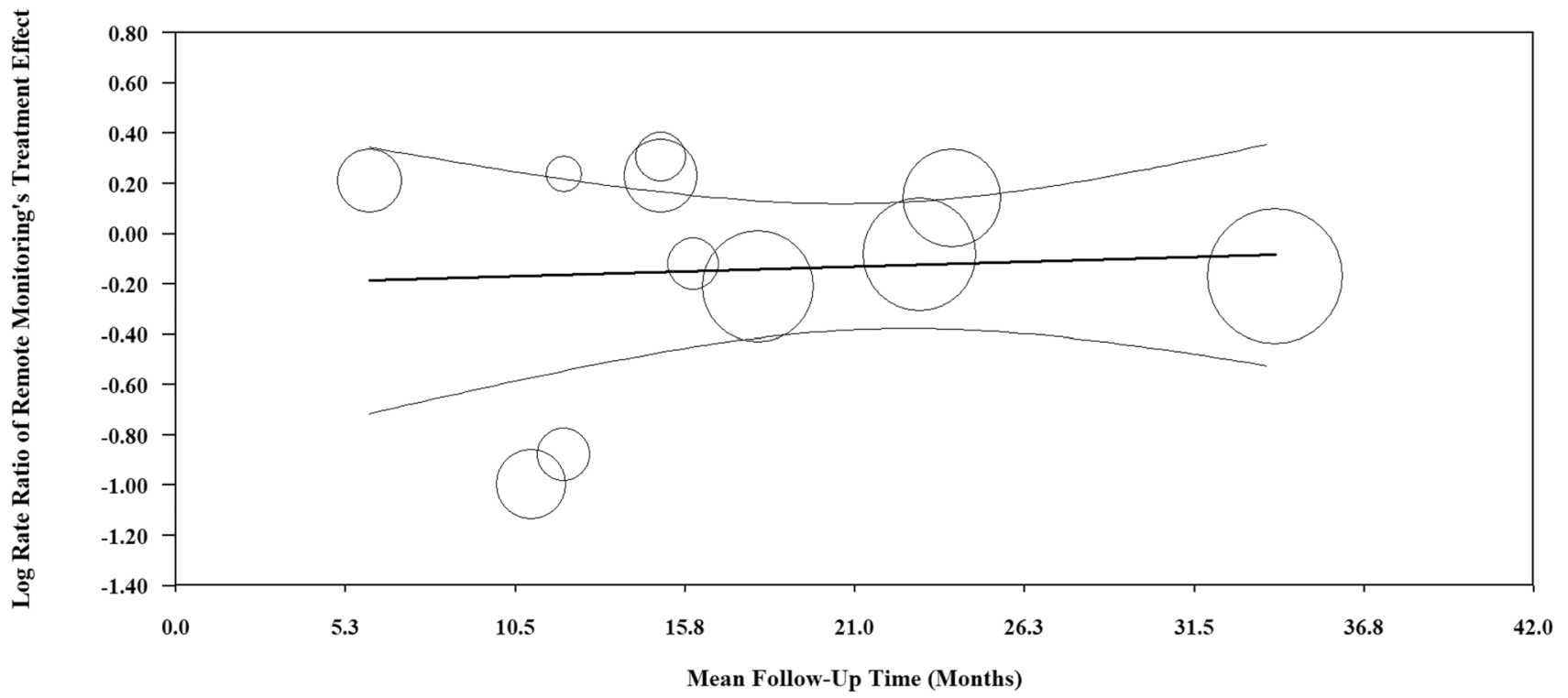

Fig. 7 This figure represents the random effect meta-regression. The log rate ratio of remote monitoring's treatment effect on mortality from each trial is plotted on the $y$-axis. The mean follow-up time in months (moderator variable) is plotted on the $x$-axis. Each circle on the graph represents an included randomized trial, and the size of the circle is proportional to the weight each study had in the regression model. The darker line in the center is the regression line and the lighter colored, outer lines represent the $95 \%$ confidence interval 
While invasive remote monitoring may not have been shown to provide high value care for HF under normal circumstance, the novel coronavirus SARS-CoV2 (COVID-19) pandemic highlighted the importance of remote monitoring of various medical conditions, including heart failure [36]. Reduced in-person visits and hesitancy to seek care in the early stages of the pandemic may have paradoxically caused a decrease in HF hospitalizations; however, this merely highlighted the need for advances in telehealth and remote monitoring for HF. Experts such as Abraham et al. have postulated that remote monitoring devices, specifically invasive devices such as CardioMEMS [16, 17], should be quickly adopted by clinicians in the absence of in-person visits [36]. This would be pertinent to aid clinicians in providing remote care and prevent further HF hospitalizations.

Interestingly, the subgroup analysis shows that implantable hemodynamic pressure monitoring, whether of RV or PA pressures [17, 21, 34], did reduce hospitalizations as compared to monitors that were centered around thoracic impedance (Fig. 5). Although thoracic impedance may be a measure of pulmonary edema, it may be limited in detecting changes in patients with chronic heart failure or may be subject to changes other heart failure, such as pneumonia. Therefore, the sensitivity and specificity of this single metric may be limited. This finding is in concordance with the findings from the CHAMPION trial, which used a regimented and aggressive treatment plan based on PA pressure-based determination of volume status [17]. This included stratifying subjects as optivolemic, hypervolemic, or hypovolemic. If volume status was optimal, no changes were made. Whereas for a designation of hypervolemia or hypovolemia, clinicians were encouraged to make immediate changes to diuretics, fluid/salt restrictions, and neurohumoral/vasodilator therapy, with close (2-3 day) follow-up often included repeating laboratory testing. This monitoring strategy proved to be effective in reducing hospitalizations.

Only one of the 11 studies had a statistically significant mortality benefit, the IN-TIME trial (RR 0.37 ; 95\% CI $0.18-0.75$ ) [23]. Notably, this study utilized a multiparameter monitoring system and is the only RCT to use such a system, although others have been studied in non-randomized prospective observational trials including PARTNERS-HF, MultiSENSE, and MANAGE-HF (NCT03237858) [18, 28, 37, 38]. Given that these studies are not RCTs (PARTNERSHF and MultiSENSE) or ongoing (MANAGE-HF), they were excluded from this analysis.

In addition, ongoing multisensory remote monitoring studies could potentially replicate the results from these implantable device studies, with the use of wearable devices measuring the same or surrogate parameters. This includes the Multisensor Monitoring in Congestive Heart Failure (MUSIC) study and the Nanosense cohort study (NCT03719079), which incorporates the third heart sound
(S3) among other metrics measured by a wearable device $[39,40]$. This would expand the number of patients that could potentially be monitored beyond those who have an indication for an ICD or CRT-D, suggesting advanced heart failure. Overall, these techniques are promising moving forward and may prove to show more benefit that intrathoracic impedance-based or intracardiac/PA pressure-based monitoring systems, with potentially less complications or side effects [11].

The results of this meta-analysis are consistent with other recent meta-analyses. Yun et al. focused on telemonitoring for HF, but not specifically using implantable devices, yet there was an all-cause mortality and HF-related mortality benefit, driven mainly by smaller clinical trials [9]. Adamson et al. showed that five clinical trials that did use hemodynamic data (intracardiac/PA pressure monitoring) had a benefit in terms of heart failure hospitalizations, which was reproduced and expanded upon in our meta-analysis [12]. A more recent meta-analysis, Alotaibi et al., of heart failure remote monitoring using implantable devices had similar conclusions to our study, however did not include the all of the RCTs due to a difference in inclusion/exclusion criteria, as well as a focus on arrhythmia-only based strategies [41].

To our knowledge, no formal meta-regression has been performed analyzing the association with follow-up time and outcomes related to HF remote monitoring. Using the mean follow-up time as the moderator on each primary endpoint, our regression analysis found no relationship between the $\log$ rate ratio of remote monitoring's effect on mortality, CV hospitalization or HF hospitalization, and mean followup time. Suggestions have been made that longer followup times would be more efficacious in detecting clinically meaningful differences in outcomes; however, this has not been proven through RCT evidence. Notably, interim results of the CHAMPION trial at 6 months showed a similar reduction in HF hospitalizations (39\%) as was seen in final study analysis at 18 months (33\% reduction) [16, 17]. This falls in line with our regression analysis on follow-up time and could serve to guide future studies.

\section{Limitations}

One limitation of this meta-analysis is the RCT heterogeneity observed among the hospitalization outcomes. The studies were conducted slightly differently, using a variety of remote monitoring devices/parameters. Based on the findings using hemodynamic pressure measurements, more studies investigating intracardiac or PA pressure monitoring may show that this specific measure is beneficial [13].

Another limitation of this meta-analysis was the outcome congruence and powering. Mortality was included in this analysis given that the data was reported in each study (and it is a valuable measure), yet it was not the primary 
outcome of the individual RCTs and these studies were not powered to detect differences in mortality. Larger enrollment and follow-up time would be required, yet this is not always the feasible given the nature of the patient $\mathrm{HF}$ severity (often NYHA classes III-IV) and including those who require implantable devices such as ICD/CRT-Ds or PA pressure monitors. In addition, not every study reported heart failure hospitalizations, with some only including cardiovascular hospitalizations (any hospitalization for heart failure, arrhythmia, myocardial infarction, etc.). Ideally, studies would report both measures (in addition to all-cause hospitalization) to determine the specific benefit of remote monitoring thereby defining reduction of types of hospitalizations, if any.

Although a large majority of the studies enrolled patients with implanted CRT-D or ICD, they each had different physiologic markers as part of their monitoring protocol. One of the 11 RCTs utilized a pulmonary artery pressure sensor [17]. Two of the 11 RCTs utilized audible patient alarms [22, 27], which adds an additional confounding factor, as these alarms notified the patients directly whenever they crossed the threshold for OptiVol fluid index of 60 (ohms). One of the 11 RCTs [26] had only initial CV hospitalizations as a primary endpoint, which would likely underreport the number of total CV hospitalizations.

\section{Future Studies}

Additional studies, especially those focused on monitoring of hemodynamic parameters, will help elucidate the role for remote monitoring. This includes the GUIDE-HF trial, a follow-up to the CHAMPION trial [17] which is poised to enroll 3600 patients in order to power for mortality [13]. A study of this magnitude would not have been feasible without initial data from CHAMPION showing a positive result. The full LAPTOP-HF trial study results will also help answer this question [42]. The study was stopped early due to device implantation adverse outcomes (during atrial septal puncture for LA pressure monitor implantation), but did have a $41 \%$ reduction in annual HF hospitalizations [43].

\section{Conclusion}

In our systematic review, meta-analysis, and meta-regression, we sought to determine whether there was evidence to suggest that implantable remote physiologic monitoring in heart failure patients results in reduced mortality and hospitalizations when compared to the standard of care. While a few individual studies showed potential benefit, our meta-analysis showed no significant difference in these outcomes between patients who underwent a remote monitoring strategy and those who had regular clinic follow-up. Given the significant morbidity and healthcare burden associated with heart failure hospitalizations, further studies should assess clinically relevant metrics that can accurately predict an exacerbation state to ideally prevent hospitalization. According to our metaanalysis, right ventricular/pulmonary pressure monitoring may reduce hospitalizations compared to impedance-based monitoring. Last, regression analysis found no relationship between mean follow-up time and primary outcomes of mortality, CV hospitalization, or HF hospitalization. In future studies, utilization of standardized remote monitoring protocols for intervention would likely allow for better assessment of the utility of heart failure remote monitoring, and possibly improve overall patient outcomes.

\section{Appendix 1 Study descriptions}

CHAMPION (Abraham, 2016) randomized 550 patients with NYHA class III HF and implanted CardioMEMS pulmonary artery pressure sensor into intervention and control groups. The intervention group $(n=270)$ had their pulmonary artery pressure readings uploaded daily and used by the investigators to guide outpatient diuretic therapy. The control group $(n=280)$ did not have their pressure readings made available to the study investigators. After mean follow-up of 6 months, the intervention group had $182 \mathrm{HF}$ hospitalizations, compared to the control group's $279 \mathrm{HF}$ hospitalizations, and 50 deaths compared to the control's 64 deaths [17].

REDUCEhf (Adamson, 2011) randomized 400 patients with class II-III HF with an implanted hemodynamic monitor system or an ICD capable of hemodynamic monitoring. The physiologic markers measured were RV systolic pressure, RV diastolic pressure, an estimate of pulmonary artery diastolic pressure (ePAD), maximum positive and negative changes in pressure over time, heart rate, and activity. The intervention group had weekly uploads of this data sent to their cardiologist, who adjusted outpatient medication regimens per their discretion, whereas the control group did not have their data made visible to their cardiologists, instead continuing standard of care phone calls from the heart failure nursing team. After a mean follow-up time of 12 months, the intervention group $(n=202)$ had 79 HF hospitalizations and 7 deaths, compared to the control group $(n=198)$ with $83 \mathrm{HF}$ hospitalizations and 9 deaths [34].

OptiLink HF (Bohm, 2016) randomized 1002 patients with class II-III $\mathrm{HF}$ and an $\mathrm{EF} \leq 35 \%$ who had recently had an ICD with or without resynchronization capability to either intervention or control group. Both groups had intrathoracic impedance measured via the OptiVol fluid index. The intervention group $(n=505)$ had alerts transmitted to the study investigators whenever a certain threshold index of intrathoracic impedance had been reached, and the physicians would then follow an intervention protocol. The 
control group $(n=507)$ did not have these alerts transmitted to the physicians, and instead continued standard of care with nursing phone calls. After a mean follow-up time of 23.6 months, the intervention group had 214 initial CV hospitalizations, of which 119 were initial HF hospitalizations, with a total of $220 \mathrm{HF}$ hospitalizations and 59 deaths. After a mean follow-up time of 22.3 months, the control group had 221 initial CV hospitalizations, of which 128 were initial HF hospitalizations, with a total of $218 \mathrm{HF}$ hospitalizations and 63 deaths [19].

MORE-CARE (Boriani, 2017) randomized $865 \mathrm{HF}$ patients with recently implanted CRT-D to either intervention or control group, where both groups' CRT-D reported automatic alerts for lung fluid accumulation (OptiVol®), atrial tachyarrhythmia (atrial tachycardia/fibrillation), and system integrity. The control group $(n=428)$ had "standard of care" defined as in-office follow-up every 4 months without any remote checks of the CRT-D alerts, whereas the intervention group $(n=437)$ had follow-up every 4 months alternating between remote checks of the CRT-D alerts and regular in-office appointments. Over the median follow-up time of 24 months, the intervention group had 197 CV hospitalizations, of which 111 were HF hospitalizations, and 40 deaths. The control group had $200 \mathrm{CV}$ hospitalizations, of which 103 were HF hospitalizations, and 34 deaths [20].

COMPASS-HF (Bourge, 2008) randomized 274 patients with NYHA class III-IV HF to either intervention $(n=134)$ or control $(n=140)$ groups. Both groups then received an implanted continuous hemodynamic monitoring device (Chronicle, Medtronic Inc.). The intervention group had physiologic data from their implanted devices reviewed weekly by a clinician, whereas the control group did not have their data reviewed during the mean follow-up time of 6 months. The intervention group had $37 \mathrm{HF}$ hospitalizations and 13 deaths, while the control group had 57 HF hospitalizations and 11 deaths [21].

LIMIT-CHF (Domenichini, 2016) randomized 80 patients with class I-III HF, EF $\leq 50 \%$, and recent implantation of an ICD or CRT-D capable of measuring the proprietary intrathoracic impedance indices from Medtronic OptiVol or SJM CorVue. The intervention group $(n=41)$ had an audible alarm set to the devices' default congestion thresholds (fluid index of 60 for OptiVol, congestion trigger of 13 for CorVue), with patients instructed to call their cardiologists if the alarm went off, and increase oral loop diuretic dose by $50 \%$ for 1 week if indices were rising. The control group $(n=39)$ did not have audible alarms set up, and instead had routine in-office follow-up. Over the median follow-up time of 375 days, the intervention group had $11 \mathrm{HF}$ hospitalizations and 4 deaths, while the control group had $6 \mathrm{HF}$ hospitalizations and 3 deaths [22].

IN-TIME (Hindricks, 2014) randomized 664 patients with class II-III HF, EF $\leq 35 \%$, and a recent dual chamber
ICD or CRT-D to either intervention $(n=333)$ or control $(n=331)$ groups. The intervention group had their hemodynamic data sent to the study investigators at a set time daily and on detection of tachyarrhythmia, and the investigators made adjustments to outpatient medication per their discretion. The control group did not have the data reviewed and instead proceeded with standard of care. Over a mean follow-up time of 335 days, the intervention group had $23 \mathrm{CV}$ hospitalizations and 10 deaths. Over a mean follow-up time of 326 days, the control group had 27 CV hospitalizations and 27 deaths [23].

EVOLVO (Landolina, 2012) randomized 200 patients with class I-III HF, EF $\leq 35 \%$, and dual chamber ICD or CRT-D capable of intrathoracic impedance monitoring to either intervention $(n=99)$ or control $(n=101)$ groups. The intervention group had data regarding thoracic impedance, arrhythmias, and ICD shocks transmitted to the study investigators, and had 4-month follow-up alternating between in-person clinic visits and remote monitoring visits based on the transmitted data, with adjustments to medications made per physicians' discretion, whereas the control group had standard of care with regular 4-month in-person clinic visits. Over the mean follow-up time of 16 months, the intervention group had $57 \mathrm{CV}$ hospitalizations and 7 deaths, whereas the control group had $49 \mathrm{CV}$ hospitalizations and 8 deaths [24].

Luthje (2015) randomized 176 patients with class I-IV HF and ICD or CRT-D to either the intervention group with remote monitoring via OptiVol alerts or a control group of standard in-office visits every 3 months. The intervention group $(n=87)$ had OptiVol alert system connected to the Medtronic CareLink Network, whereas the control group $(n=89)$ did not have their devices connected to the network, and instead had regular in-office visits. Of note, OptiVol audible alerts were disabled in both groups. Both groups were followed for 15 months. The intervention group had $20 \mathrm{HF}$ hospitalizations during that follow-up time, whereas the control group had $22 \mathrm{HF}$ hospitalizations. Eight patients died in the intervention group, compared to 6 patients who died in the control group [25].

REM-HF (Morgan, 2017) randomized 1650 patients with NYHA class II-IV HF and implanted ICD to either intervention $(n=824)$ or control $(n=826)$ groups. The intervention protocol consisted of weekly uploads of thoracic impedance, $\mathrm{Bi}-\mathrm{V}$ pacing, HR variability, arrhythmia, and AF/AT burden, with guide book-directed adjustment of medical therapy by a designated clinician, whereas the control group had standard of care phone calls and clinic visits. Over a median followup of 34 months, the intervention group had 315 initial CV hospitalizations and 128 deaths, while the control group had 297 initial CV hospitalizations and 152 deaths [26].

DOT-HF (Van Veldhuisen, 2011) randomized 335 patients with NYHA class II-IV HF, EF $\leq 35 \%$, and implanted ICD 
or CRT-D capable of thoracic impedance monitoring with the OptiVol system to either intervention $(n=168)$ or control $(n=167)$ groups. The intervention group had an audible alarm set for an OptiVol fluid congestion threshold, with patients instructed to call the study investigators when the alarms went off, at which point interventions were performed per clinicians' discretion. The control group did not have any audible alarms set for a specific threshold. Over a mean follow-up time of 15 months, the intervention group had 115 CV hospitalizations, of which 60 were HF hospitalizations, as well as 19 deaths. The control group had 74 CV hospitalizations, of which 36 were HF hospitalizations, and 15 deaths [27].

Supplementary Information The online version contains supplementary material available at https://doi.org/10.1007/s10741-021-10150-5.

Acknowledgements Amy Knehans performed the initial search for the systematic review. We thank Dr. Omaima Ai for discussions regarding heart failure remote monitoring.

Author contribution AH designed the research study; AH and SM performed the systematic review and analyzed the data for the metaanalysis; $\mathrm{MN}$ analyzed the data for the meta-regression; $\mathrm{AH}$ and SM wrote the paper; $\mathrm{MN}, \mathrm{AD}$, and JB edited the paper and contributed to the discussion.

Funding This work was supported by the 2019-2020 Penn State Internal Medicine House Staff Award granted to A. Hajduczok.

Availability of data and material Primary data can be requested from the corresponding author.

\section{Declarations}

Conflict of interest John Boehmer: Boston Scientific-consultant, speaker, research grants; Nanowear, Inc.-consulting and research grant; Medtronic - consulting; Abbott-research grant. All other authors: none.

\section{References}

1. Yancy CW, Jessup M, Bozkurt B, Butler J, Casey DE Jr, Colvin MM, Drazner MH, Filippatos GS, Fonarow GC, Givertz MM, Hollenberg SM, Lindenfeld J, Masoudi FA, McBride PE, Peterson PN, Stevenson LW, Westlake C (2017) 2017 ACC/AHA/HFSA Focused Update of the 2013 ACCF/AHA guideline for the management of heart failure: a report of the American College of Cardiology/American Heart Association Task Force on Clinical Practice Guidelines and the Heart Failure Society of America. J Card Fail 23(8):628-651

2. Ponikowski P, Voors A, Anker SD, Bueno H, Cleland JG, Coats AJ, Falk V, González-Juanatey JR, Harjola VP, Jankowska EA, Jessup M, Linde C, Nihoyannopoulos P, Parissis JT, Pieske B, Riley JP, Rosano GM, Ruilope LM, Ruschitzka F, Rutten FH, van der Meer P (2016) 2016 ESC Guidelines for the diagnosis and treatment of acute and chronic heart failure: the task force for the diagnosis and treatment of acute and chronic heart failure of the European Society of Cardiology (ESC) Developed with the special contribution of the Heart Failure Association (HFA) of the ESC. Eur J Heart Fail 18(8):891-975

3. Ambrosy AP, Fonarow GC, Butler J, Chioncel O, Greene SJ, Vaduganathan M, Nodari S, Lam CS, Sato N, Shah AN, Gheorghiade M (2014) The global health and economic burden of hospitalizations for heart failure: lessons learned from hospitalized heart failure registries. J Am Coll Cardiol 63(12):1123-1133

4. Lee KK, Yang J, Hernandez AF, Steimle AE, Go AS (2016) Postdischarge follow-up characteristics associated with 30-day readmission after heart failure hospitalization. Med Care 54(4):365

5. Dickinson MG, Allen LA, Albert NA, DiSalvo T, Ewald GA, Vest AR, Whellan DJ, Zile MR, Givertz MM (2018) Remote monitoring of patients with heart failure: a white paper from the Heart Failure Society of America Scientific Statements Committee. J Card Fail 24(10):682-694

6. Whellan DJ, Sarkar S, Koehler J, Small RS, Boyle A, Warman EN, Abraham WT (2013) Development of a method to risk stratify patients with heart failure for 30-day readmission using implantable device diagnostics. Am J Cardiol 111(1):79-84

7. Desai AS, Stevenson LW (2010) Connecting the circle from home to heart-failure disease management. New Engl J Med 363(24):2364-2367

8. Chaudhry SI, Mattera JA, Curtis JP, Spertus JA, Herrin J, Lin Z, Phillips CO, Hodshon BV, Cooper LS, Krumholz HM (2010) Telemonitoring in patients with heart failure. New Engl J Med 363(24):2301-2309

9. Yun JE, Park JE, Park HY, Lee HY, Park DA (2018) Comparative effectiveness of telemonitoring versus usual care for heart failure: a systematic review and meta-analysis. J Card Fail 24(1):19-28

10. Klersy C, Boriani G, De Silvestri A, Mairesse GH, Braunschweig F, Scotti V, Balduini A, Cowie MR, Leyva F, Health Economics Committee of the European Heart Rhythm Association (2016) Effect of telemonitoring of cardiac implantable electronic devices on healthcare utilization: a meta-analysis of randomized controlled trials in patients with heart failure. Eur J Heart Fail 18(2):195-204

11. Ali O, Hajduczok AG, Boehmer JP (2020) Remote physiologic monitoring for heart failure. Curr Cardiol Rep 22(8):68

12. Adamson PB, Ginn G, Anker SD, Bourge RC, Abraham WT (2017) Remote haemodynamic-guided care for patients with chronic heart failure: a meta-analysis of completed trials. Eur J Heart Fail 19(3):426-433

13. Lindenfeld J, Abraham W, Maisel A, Zile M, Smart F, Costanzo MR, Mehra MR, Ducharme A, Sears SF, Desai AS, Paul S, Sood P, Johnson N, Ginn G, Adamson PB (2019) HemodynamicGUIDEd management of heart failure (GUIDE-HF). Am Heart J 214:18-27

14. Jermyn R, Alam A, Kvasic J, Saeed O, Jorde U (2017) Hemodynamicguided heart-failure management using a wireless implantable sensor: infrastructure methods and results in a community heart failure disease-management program. Clin Cardiol 40(3):170-176

15. Zile MR, Bennett TD, St. John Sutton M, Cho YK, Adamson PB, Aaron MF, Aranda JM Jr, Abraham WT, Smart FW, Stevenson LW, Kueffer FJ, Bourge RC (2008) Transition from chronic compensated to acute decompensated heart failure: pathophysiological insights obtained from continuous monitoring of intracardiac pressures. Circulation 118(14):1433-1441

16. Abraham WT, Adamson PB, Bourge RC, Aaron MF, Costanzo MR, Stevenson LW, Strickland W, Neelagaru S, Raval N, Krueger S, Weiner S, Shavelle D, Jeffries B, Yadav JS, CHAMPION Trial Study Group (2011) Wireless pulmonary artery haemodynamic monitoring in chronic heart failure: a randomised controlled trial. Lancet 377(9766):658-666 
17. Abraham WT, Stevenson LW, Bourge RC, Lindenfeld JA, Bauman JG, Adamson PB, CHAMPION Trial Study Group (2016) Sustained efficacy of pulmonary artery pressure to guide adjustment of chronic heart failure therapy: complete followup results from the CHAMPION randomised trial. Lancet 387(10017):453-461

18. Boehmer JP, Hariharan R, Devecchi FG, Smith AL, Molon G, Capucci A, An Q, Averina V, Stolen CM, Thakur PH, Thompson JA, Wariar R, Zhang Y, Singh JP (2017) A multisensor algorithm predicts heart failure events in patients with implanted devices: results from the MultiSENSE study. JACC Heart Fail 5(3):216-225

19. Böhm M, Drexler H, Oswald H, Rybak K, Bosch R, Butter C, Klein G, Gerritse B, Monteiro J, Israel C, Bimmel D, Käab S, Huegl B, Brachmann J, OptiLink HF Study Investigators (2016) Fluid status telemedicine alerts for heart failure: a randomized controlled trial. Eur Heart J 37(41):3154-3163

20. Boriani G, Da Costa A, Quesada A, Ricci RP, Favale S, Boscolo G, Clementy N, Amori V, Mangoni di S Stefano L, Burri H, MORECARE Study Investigators (2016) Effects of remote monitoring on clinical outcomes and use of healthcare resources in heart failure patients with biventricular defibrillators: results of the MORECARE multicentre randomized controlled trial. Eur J Heart Fail 19(3):416-425

21. Bourge RC, Abraham WT, Adamson PB, Aaron MF, Aranda JM Jr, Magalski A, Zile MR, Smith AL, Smart FW, O'Shaughnessy MA, Jessup ML, Sparks B, Naftel DL, Stevenson LW, COMPASS-HF Study Group (2008) Randomized controlled trial of an implantable continuous hemodynamic monitor in patients with advanced heart failure: the COMPASS-HF study. J Am Coll Cardiol 51(11):1073-1079

22. Domenichini G, Rahneva T, Diab IG, Dhillon OS, Campbell NG, Finlay MC, Baker V, Hunter RJ, Earley MJ, Schilling RJ (2016) The lung impedance monitoring in treatment of chronic heart failure (the LIMIT-CHF study). Europace 18(3):428-435

23. Hindricks G, Taborsky M, Glikson M, Heinrich U, Schumacher B, Katz A, Brachmann J, Lewalter T, Goette A, Block M, Kautzner J, Sack S, Husser D, Piorkowski C, Søgaard P, IN-TIME study group (2014) Implant-based multiparameter telemonitoring of patients with heart failure (IN-TIME): a randomised controlled trial. Lancet 384(9943):583-590

24. Landolina M, Perego GB, Lunati M, Curnis A, Guenzati G, Vicentini A, Parati G, Borghi G, Zanaboni P, Valsecchi S, Marzegalli M (2012) Remote monitoring reduces healthcare use and improves quality of care in heart failure patients with implantable defibrillators: the evolution of management strategies of heart failure patients with implantable defibrillators (EVOLVO) study. Circulation 125(24):2985-2992

25. Lüthje L, Vollmann D, Seegers J, Sohns C, Hasenfuß G, Zabel M (2015) A randomized study of remote monitoring and fluid monitoring for the management of patients with implanted cardiac arrhythmia devices. Europace 17(8):1276-1281

26. Morgan JM, Kitt S, Gill J, McComb JM, Ng GA, Raftery J, Roderick P, Seed A, Williams SG, Witte KK, Wright DJ, Harris S, Cowie MR (2017) Remote management of heart failure using implantable electronic devices. Eur Heart J 38(30):2352-2360

27. van Veldhuisen DJ, Braunschweig F, Conraads V, Ford I, Cowie MR, Jondeau G, Kautzner J, Aguilera RM, Lunati M, Yu CM, Gerritse B, Borggrefe M, Investigators DOT-HF (2011) Intrathoracic impedance monitoring audible patient alerts and outcome in patients with heart failure. Circulation 124(16):1719-1726

28. Whellan DJ, Ousdigian KT, Al-Khatib SM, Pu W, Sarkar S, Porter CB, Pavri BB, O'Connor CM, PARTNERS Study Investigators (2010) Combined heart failure device diagnostics identify patients at higher risk of subsequent heart failure hospitalizations: results from PARTNERS HF (Program to Access and Review Trending Information and Evaluate Correlation to Symptoms in Patients With Heart Failure) study. J Am Coll Cardiol 55(17):1803-1810

29. Liberati A, Altman DG, Tetzlaff J, Mulrow C, Gøtzsche PC, Ioannidis JP, Clarke M, Devereaux PJ, Kleijnen J, Moher D (2009) The PRISMA statement for reporting systematic reviews and meta-analyses of studies that evaluate health care interventions: explanation and elaboration. PLoS Med 6(7)

30. Moher D, Liberati A, Tetzlaff J, Altman DG, Prisma Group (2009) Preferred reporting items for systematic reviews and meta-analyses: the PRISMA statement. PLoS Med 6(7):e1000097

31. Higgins JP, Altman DG, Gøtzsche PC, Jüni P, Moher D, Oxman AD, Savovic J, Schulz KF, Weeks L, Sterne JA, Cochrane Bias Methods Group, Cochrane Statistical Methods Group (2011) The Cochrane Collaboration's tool for assessing risk of bias in randomised trials. BMJ 18(343)

32. Sterne JAC, Savović J, Page MJ, Elbers RG, Blencowe NS, Boutron I, Cates CJ, Cheng HY, Corbett MS, Eldridge SM, Emberson JR, Hernán MA, Hopewell S, Hróbjartsson A, Junqueira DR, Jüni P, Kirkham JJ, Lasserson T, Li T, McAleenan A, Reeves BC, Shepperd S, Shrier I, Stewart LA, Tilling K, White IR, Whiting PF, Higgins JPT (2019) RoB 2: a revised tool for assessing risk of bias in randomised trials. BMJ 366(4898)

33. Higgins JP, Thompson SG (2002) Quantifying heterogeneity in a meta-analysis. Stat Med 21(11):1539-1558

34. Adamson PB, Gold MR, Bennett T, Bourge RC, Stevenson LW, Trupp R, Stromberg K, Wilkoff BL, Costanzo MR, Luby A, Aranda JM, Heywood JT, Baldwin HA, Aaron M, Smith A, Zile M (2011) Continuous hemodynamic monitoring in patients with mild to moderate heart failure: results of The Reducing Decompensation Events Utilizing Intracardiac Pressures in Patients With Chronic Heart Failure (REDUCEhf) trial. Congest Heart Fail 17(5):248-254. https://doi.org/10.1111/j.1751-7133.2011.00247.x

35. Parmar MK, Torri V, Stewart L (1998) Extracting summary statistics to perform meta-analyses of the published literature for survival endpoints. Stat Med 17(24):2815-2834

36. Abraham WT, Fiuzat M, Psotka MA, O'Connor CM (2020) Heart failure collaboratory statement on remote monitoring and social distancing in the landscape of COVID-19. JACC Heart Fail 8(8):692-694

37. Cowie MR, Sarkar S, Koehler J, Whellan DJ, Crossley GH, Tang WH, Abraham WT, Sharma V, Santini M (2013) Development and validation of an integrated diagnostic algorithm derived from parameters monitored in implantable devices for identifying patients at risk for heart failure hospitalization in an ambulatory setting. Eur Heart J 34(31):2472-2480

38. NCT03237858 (2021) Multiple Cardiac Sensors for the Management of Heart Failure (MANAGE-HF). https://clinicaltrials.gov/ ct2/show/NCT03237858

39. NCT03719079 (2020) Nanowear Heart Failure Management Multi-sensor Algorithm (Nanosense). https://clinicaltrials.gov/ ct2/show/NCT03719079

40. Anand IS, Tang WW, Greenberg BH, Chakravarthy N, Libbus I, Katra RP (2012) Design and performance of a multisensor heart failure monitoring algorithm: results from the multisensor monitoring in congestive heart failure (MUSIC) study. J Card Fail 18(4):289-295

41. Alotaibi S, Hernandez-Montfort J, Ali OE, El-Chilali K, Perez BA (2020) Remote monitoring of implantable cardiac devices in heart failure patients: a systematic review and meta-analysis of randomized controlled trials. Heart Fail Rev 25(3):469-479

42. Maurer MS, Adamson PB, Costanzo MR, Eigler N, Gilbert J, Gold MR, Klapholz M, Saxon LA, Singh JP, Troughton R, Abraham WT 
(2015) Rationale and design of the left atrial pressure monitoring to optimize heart failure therapy study (LAPTOP-HF). J Card Fail 21(6):479-488

43. Abraham WT, Adamson PB, Costanzo MR, Eigler N, Gold M, Klapholz M, Maurer M, Saxon L, Singh J, Troughton R (2016)
Hemodynamic monitoring in advanced heart failure: results from the LAPTOP-HF trial. J Card Fail 22(11):940

Publisher's Note Springer Nature remains neutral with regard to jurisdictional claims in published maps and institutional affiliations.

\section{Authors and Affiliations}

\section{Alexander G. Hajduczok ${ }^{1}$ (1) - Samer N. Muallem ${ }^{1} \cdot$ Matthew S. Nudy $^{2} \cdot$ Ami L. DeWaters $^{1} \cdot$ John P. Boehmer ${ }^{2}$}

Alexander G. Hajduczok

alexander.hajduczok@jefferson.edu

Samer N. Muallem

smuallem@pennstatehealth.psu.edu

Matthew S. Nudy

mnudy@pennstatehealth.psu.edu

Ami L. DeWaters

adewaters@pennstatehealth.psu.edu
John P. Boehmer

jboehmer@pennstatehealth.psu.edu

1 Division of Internal Medicine, Penn State Health Milton S. Hershey Medical Center, Penn State College of Medicine, 500 University Drive, Hershey, PA 17033, USA

2 Division of Cardiology, Penn State Health Milton S. Hershey Medical Center, Penn State College of Medicine, 500 University Drive, PA H047, 17033 Hershey, USA 